Rogério Lessa Horta'

(D) https://orcid org/0000-0001-5195-8014

Eduardo Guimarães Camargo² Ohtps:///orid.org/0000-0002-5404-2682

Marcus Levi Lopes Barbosa ${ }^{3}$

Onttps://orcid.org/0000-0001-5413-8695

Pedro José Sartorelli Lantin² Ohttps://orcid.org/0000-0003-2354-2411

Talia Greici Sette ${ }^{2}$

Ohttps://orcid.org/0000-0002-4478-0761

Thaís Caroline Guedes Lucini

(1) https://orcid org/0000-0001-8474-5875

Aline Faria Silveira ${ }^{2}$

Ohttps://orid.org/0000-0002-4728-5715

Lizziê Zanini²

Onttps://orcid.org/0000-0002-0045-0277

Bibiana Andrade Lutzky²

Ohttps://orcid.org/0000-0002-3595-4037

\section{O estresse e a saúde mental de profissionais da linha de frente da COVID-19 em hospital geral}

\author{
Front line staff stress and mental health during \\ COVID-19 pandemic in a general hospital
}

DOI: $10.1590 / 0047-2085000000316$

\begin{abstract}
RESUMO
Objetivo: Investigar os efeitos da atuação na linha de frente da COVID-19 na saúde mental de profissionais de hospital público. Métodos: Análise transversal de entrevistas de ingresso em estudo prospectivo, com abordagem mista, em hospital da rede pública no Sul do Brasil. Resultados: Foram entrevistadas 123 pessoas, 76\% profissionais de enfermagem e 81\% mulheres. Escore igual ou superior a sete pontos no Self-Reporting Questionnaire (SRQ-20) foi obtido para 40\% da amostra, 45\% tiveram escore igual ou superior a 25 pontos na Perceived Stress Scale (PSS) e 41\% atingiram escores compatíveis com burnout no Inventário de Burnout de Oldenburg (OBI). Os desfechos estiveram associados entre si $(p<0,05)$, mas nenhuma associação foi verificada com as variáveis independentes. Nas entrevistas em profundidade, foram destacados como dificuldades: longos plantões sem intervalos, bem como paramentação, pressão e cansaço maiores que os habituais, isolamento no próprio hospital, risco da própria contaminação e temores e culpa relacionados às famílias. A união da equipe apareceu como aspecto que favorece o desempenho no enfrentamento dessa situação. Conclusões: Profissionais apresentam quadro de sofrimento psicossocial. Recomenda-se priorizar repouso e intervalos, o que poderá exigir adequações de rotinas e espaços físicos, além de ampliar a oferta de apoio emocional às equipes.
\end{abstract}

\section{PALAVRAS-CHAVE}

COVID-19, hospital, pandemia, estresse, equipe.

\section{ABSTRACT}

Objective: To investigate psychosocial effects on health professionals from the frontline activity in the pandemic. Methods: Cross-sectional analysis of entry interviews in a prospective study about the stress and mental health of COVID-19 frontline workers in a public hospital in Southern Brazil. The study has a mixed approach. Results: 123 people interviewed, 76\% were nursing professionals and $81 \%$ women. A score of seven or more on the Self-Reporting Questionnaire (SRQ-20) was obtained for $40 \%$ of the sample, $45 \%$ had a score of 25 or more on the Perceived Stress Scale (PSS) and $41 \%$ achieved scores compatible with burnout in the Oldenburg Burnout Inventory (OBI). The outcomes were associated with each other $(p<0.05)$, but no association was found with the independent variables. Long shifts without breaks due to vestments, pressure and fatigue greater than usual, isolation in the hospital itself, risk of contamination itself and fears and guilt related to families were highlighted in the in-depth interviews. The team unity appeared as an aspect that favors performance in COVID-19 coping. Conclusions: Professionals are suffering and it is recommended to prioritize rest and breaks, which may require adjustments to routines and physical spaces, in addition to expanding the offer of emotional support to the teams of health workers.

\section{KEYWORDS}

COVID-19, hospital, pandemic, stress, team.
Received in: Jan/13/2021. Approved in: Feb/10/2021

1 Universidade Feevale, Curso de Medicina e Mestrado Acadêmico em Psicologia, Novo Hamburgo, RS, Brasil.

2 Universidade Feevale, Curso de Medicina, Novo Hamburgo, RS, Brasil.

3 Universidade Feevale, Curso de Psicologia e Mestrado Acadêmico em Psicologia, Novo Hamburgo, RS, Brasil.

Address for correspondence: Rogério Horta. Rua Jary, 671, ap. 703, Passo d'Areia - 91350-170 - Porto Alegre, RS, Brasil. E-mail: rogeriohortamed@gmail.com 


\section{INTRODUÇÃO}

Profissionais da saúde parecem vulneráveis aos efeitos psicossociais da pandemia de COVID-19 ${ }^{1-5}$. Quem atua diretamente na assistência está no que se convencionou chamar de linha de frente da pandemia. Como fontes de estresse e sobrecarrega, são apontadas as seguintes condições: natureza da própria infecção; testes insuficientes; falta de vacinas ou de um tratamento eficaz; evolução grave de alguns pacientes; falta de equipamentos de proteção individual (EPI) e de suprimentos médicos; cargas de trabalho prolongadas; condições inadequadas de repouso ${ }^{5,6}$.

O cuidado com profissionais da saúde passa por garantir o acesso continuado à informação clínico-epidemiológica ou a ambientes adequados de descanso dentro das unidades, além do monitoramento do estresse, com garantia de assistência adequada e intervenção profissional em saúde mental sempre que indicado ${ }^{6-8}$.

O Hospital Municipal de Novo Hamburgo (HMNH) é referência da rede pública de saúde para o atendimento de pacientes com COVID-19 na região do Vale do Sinos, no Sul do Brasil. Ali estão envolvidos profissionais de diferentes áreas, expostos aos estressores psicossociais inerentes à pandemia.

Acessar a saúde mental desses profissionais e seu nível de percepção de estresse durante a vigência deste período crítico é essencial para a identificação de condições facilitadoras do cumprimento de seu papel e preservação de sua saúde, bem como de condições desfavoráveis, uma vez que pouco ainda é conhecido nesse sentido sobre a COVID-19.

Um estudo prospectivo acompanhou esse grupo de profissionais durante o primeiro período crítico da pandemia nessa região, de junho a outubro de 2020, permitindo acompanhar as variações de níveis de estresse e sofrimento no enfrentamento da pandemia, além de eventual detecção precoce e encaminhamento para tratamento dos quadros clínicos decorrentes da exposição ocupacional.

Este artigo apresenta dados de um recorte transversal com a análise das entrevistas de ingresso no estudo, refletindo as condições em que as equipes do hospital foram encontradas pelos pesquisadores. Seus achados são relevantes e originais ao retratarem condições efetivamente manifestas por equipes atuando no enfrentamento e podem contribuir para a construção de políticas no setor. É objetivo do estudo investigar os efeitos da atuação na linha de frente da COVID-19 na saúde mental de profissionais de hospital público.

\section{MÉTODOS}

Trata-se de recorte transversal de estudo prospectivo, com abordagem mista, envolvendo profissionais da saúde que atendem pacientes com COVID-19 em hospital da rede pública de saúde. Os participantes foram informados do estudo pela direção do hospital e por visitas dos pesquisadores.
Seus contatos, fornecidos pelo hospital, permitiram acesso por chamada telefônica ou via aplicativos de voz ou mensagens. Todos receberam o Termo de Consentimento Livre e Esclarecido (TCLE), que foi lido e reenviado assinado. Opcionalmente, foi oferecida a confirmação de concordância em participar no estudo em manifestação por e-mail ou no próprio aplicativo, formalizando ter lido e concordar com o TCLE.

Foram relacionadas como profissionais em linha de frente 278 pessoas. Todas foram procuradas. Sempre que não se conseguia contato, tentativas foram repetidas em dias e horários distintos. Contatos não efetivados foram informados à coordenação, que fez pelo menos mais duas tentativas. Foi, também, buscada junto ao hospital a confirmação dos dados.

Foram excluídas 112 pessoas (40\%), das quais 80 (72\%) informaram não atuar em linha de frente ou já não estar mais vinculadas ao hospital, evidenciando grande rotatividade. Uma técnica de enfermagem não alcançada no período faleceu pela COVID-19 naquele período. Apesar de elevado, o número de exclusões acompanha a indicação do hospital da necessidade de apresentar relação ampliada por afastamentos e desligamentos, especialmente com o rápido crescimento do número de casos nas semanas em que as entrevistas foram iniciadas. Das 166 pessoas efetivamente passíveis de inclusão no estudo, 43 (26\%) formalizaram recusa em participar, com cerca de dois terços delas referindo sobrecarga e muito cansaço ou por estarem doentes ou com familiar adoecido.

Ingressaram no estudo 123 pessoas. Uma comparação de proporções pelo teste do qui-quadrado de Pearson para distribuição das pessoas consideradas potenciais sujeitos do estudo e as que responderam às entrevistas de ingresso segundo a atividade profissional exercida $(p=0,6886)$ e o sexo $(p=0,8238)$ não evidenciou diferença significativa.

Eram critérios de inclusão no estudo: ser profissional da linha de frente da COVID-19 no hospital, estar em atividade no período de desenvolvimento do estudo, concordar em participar e assinar o TCLE. Eram critérios de exclusão: não estar acessível por nenhum dos meios propostos para entrevista a distância ou apresentar impedimento por condição clínica de qualquer ordem ou outro impedimento pessoal.

A coleta dos dados ocorreu em meio à forte inclinação das curvas com elevação do número de casos, hospitalizações e óbitos, tanto no estado quanto no município. As entrevistas de ingresso foram realizadas no intervalo de 11 de junho a 14 de agosto de 2020. O período correspondeu ao intervalo entre as Semanas Epidemiológicas (SE) 24 a 33. Na SE 24, eram registrados, no Rio Grande do Sul, 16.258 casos, 2.190 hospitalizações e 350 óbitos por COVID-19, e o município de Novo Hamburgo registrava 12,3 hospitalizações por 100 mil habitantes e uma taxa de mortalidade de 2,0 óbitos a 
cada 100 mil habitantes 9 . Até a SE 33, eram contados 22.456 casos, 10.406 hospitalizações e 2.713 óbitos no estado e 105 hospitalizações a cada 100 mil habitantes e 26,1 óbitos a cada 100 mil habitantes no município, entre as mais altas do estado $^{10}$. Esse intervalo é tido como o maior pico na região, com estabilização identificada a partir de seu final ${ }^{10}$.

As entrevistas iniciais, realizadas com 123 profissionais da linha de frente da COVID-19, foram aplicadas por equipe previamente treinada e se caracterizavam por breve inquérito que permitiu a coleta de dados objetivos com base em instrumento padronizado, com as variáveis relacionadas a seguir. Durante a etapa das entrevistas iniciais, 10 profissionais aceitaram, também, falar livremente sobre suas vivências no serviço, compondo a dimensão qualitativa do estudo, com entrevistas em profundidade.

São desfechos de interesse na dimensão quantitativa: escore no Self-Reporting Questionnaire (SRQ-20) igual ou superior a sete pontos, indicativo de ocorrência de transtornos mentais comuns ${ }^{11}$; escore no PSS (Perceived Stress Scale) ${ }^{12}$ equivalente ou superior a 25 pontos, que é considerado percepção de estresse moderada ou alta13; e escore médio elevado nas duas dimensões do OBI (Oldenburg Burnout Inventory), definido como pontuação igual ou superior a 2,25 para a dimensão Exaustão e 2,1 para a dimensão Distanciamento do Trabalho, correspondendo à ocorrência de burnout ${ }^{14}$.

As covariáveis, em polo distal, segundo o modelo teórico proposto, são variáveis demográficas (sexo, idade, residir em Novo Hamburgo ou fora, ter cônjuge, viver só ou não e escolaridade). Em posição intermediária, estão as variáveis relacionadas ao trabalho (semana de ingresso no estudo, atividade profissional, tempo de exercício profissional, tempo de vínculo na instituição, carga horária efetiva na totalidade dos vínculos profissionais e carga horária efetivamente realizada na semana que antecedeu a entrevista em linha de frente). Em polo proximal aos desfechos, estão as variáveis relacionadas à saúde (ter sintomas e ter feito testagem para COVID-19, estar ou não em atendimento psicoterápico ou em uso de psicofármaco e uso referido de substâncias). As variáveis contínuas ou discretas foram agrupadas e categorizadas segundo a mediana de sua distribuição.

As entrevistas foram gravadas e os dados foram registrados no Google Forms, que permitiu a coleta em interface amigável e salvamento instantâneo. As opções de respostas eram apresentadas no dispositivo, minorando erros de digitação dos dados, com salvamento de cópias em nuvem. Os registros em áudio permitiram verificação de inconsistências e correção de eventuais erros de digitação.

Uma rodada de entrevistas com profissionais de saúde voluntários, não integrantes das equipes, foi realizada de forma que toda a equipe realizasse a rotina completa após treinamento com exercícios de role-play. Os dados coletados nessa etapa serviram para ajustes e reformulações nos procedimentos, instrumentos e banco de dados, não sendo considerados para análises posteriores.

A coordenação do estudo fez contato com 10\% dos entrevistados incluídos no estudo, para controle de qualidade e confirmação da realização das entrevistas, checando a veracidade dos dados pela repetição de uma questão objetiva. A verificação de consistência e a análise dos dados foram realizadas em software SPSS 22.0, para o qual as planilhas foram convertidas.

Foram estimadas frequências absolutas e relativas dos desfechos e covariáveis, assim como as razões de prevalência dos desfechos de interesse por regressão de Poisson com variância robusta em análises bivariável e ajustada. Foram incluídas nas análises ajustadas variáveis associadas aos desfechos até a significância de 20\% ( $p \leq 0,20)$ em três modelos: o primeiro com as variáveis demográficas, o segundo com as do modelo anterior mais as variáveis relacionadas às condições de trabalho e o terceiro com as anteriores mais as variáveis relacionadas à saúde dos profissionais. O nível de significância de 5\% ( $p \leq 0,05)$ foi considerado significativo.

A questão inicial na entrevista em profundidade era: "- Gostaria que me contasse como está sendo sua experiência neste período atuando na linha de frente no hospital". Foram aplicadas as seguintes questões norteadoras ao longo das entrevistas "- O que tem ajudado neste período?" e "- 0 que tem atrapalhado neste período?".

As entrevistas foram gravadas na íntegra, salvas em arquivo de áudio e, depois, transcritas. Cada uma delas foi lida na íntegra para uma compreensão geral da narrativa e submetida à análise argumentativa. O objetivo da análise argumentativa é condensar uma grande quantidade de material de forma que permita a análise sem perda da essência original ${ }^{15}$. Foram levadas em consideração as partes do argumento: dados, proposição, garantia, apoio e refutação, sintetizando os pontos principais em cada parágrafo, parafraseando o menos possível e verificando, por releitura ou revisão por terceiro, a sua fidedignidade. O mesmo foi feito sistematicamente para todo o material em análise, permitindo constituir um mapa que destaca as principais proposições encontradas, com os respectivos conjuntos de dados, apoios ou garantias e refutações reconhecidos. A análise dos dados tem base descritiva, com orientação sistêmica, voltada à compreensão das relações entre elementos que ajudam a evidenciar o que ajuda e o que dificulta a atuação em linha de frente. A discussão dos dados é baseada na estratégia de triangulação concomitante dos dados quanti e qualitativos ${ }^{16}$.

A investigação obedece a critérios da Resolução no 466/2012 do Conselho Nacional de Saúde. O projeto foi aprovado no Numesc (Núcleo Municipal de Educação em Saúde Coletiva) de Novo Hamburgo e pelo Comitê de Ética em Pesquisa (CEP) da Universidade Feevale, com Parecer n4.070.041, de 4 dejunho de 2020, evem sendo desenvolvido 
com recursos próprios dos pesquisadores e da Universidade Feevale. O estudo é baseado na rede de serviços de saúde do município de Novo Hamburgo. Eventuais necessidades de atendimento detectadas têm sido encaminhadas a serviço da rede de saúde local ou serviços voluntários identificados.

\section{RESULTADOS}

A Tabela 1 apresenta a distribuição de respondentes segundo os desfechos do estudo, em que $40 \%$ das pessoas referiram escores compatíveis com transtornos mentais comuns no SRQ-20 e 45\% tiveram escores iguais ou superiores a 25 pontos no PSS. No OBI, 60\% atingiram escores de exaustão e 49\% apresentaram distanciamento do trabalho, indicando dificuldade de manter a atenção e a dedicação plenas às atividades. Burnout estava presente para $41 \%$ do grupo.

Tabela 1. Prevalência estimada dos desfechos nas entrevistas de ingresso - Novo Hamburgo, 2020 ( $N=123)$

\begin{tabular}{|c|c|c|c|}
\hline Variável & Categoria & $\mathbf{n}$ & $\%$ \\
\hline \multicolumn{4}{|c|}{ Escore no SRQ-20 } \\
\hline & Até 7 pontos & 74 & 60 \\
\hline & 7 pontos ou mais & 49 & 40 \\
\hline \multicolumn{4}{|c|}{ Estresse Percebido - PSS } \\
\hline & Até 24 pontos & 67 & 55 \\
\hline & 25 pontos ou mais & 56 & 45 \\
\hline \multicolumn{4}{|c|}{ Exaustão no OBI } \\
\hline & Não & 49 & 40 \\
\hline & $\operatorname{Sim}$ & 74 & 60 \\
\hline \multicolumn{4}{|c|}{ Distanciamento no OBI } \\
\hline & Não & 63 & 51 \\
\hline & $\operatorname{Sim}$ & 60 & 49 \\
\hline \multicolumn{4}{|c|}{ Burnout no OBI (exaustão + distanciamento) } \\
\hline & Não & 73 & 59 \\
\hline & $\operatorname{Sim}$ & 50 & 41 \\
\hline
\end{tabular}

SRQ-20: Self-Reporting Questionnaire; PSS: Perceived Stress Scale; OBI: Oldendurg Burnout Inventory.

A ocorrência de qualquer desfecho aumenta a possibilidade de ocorrência dos demais. Foram estimadas razões de prevalência (RP) de percepção de estresse no PSS $\geq 25$ pontos, em análise bivariável, com escore igual ou superior a 7 no SRQ-20 de 1:3,776 [intervalo de confiança (IC) de 95\%: 2,114-6,742], tendo escore menor que 7 como referência. Para burnout com escore igual ou superior a 7 no SRQ-20, foi obtida RP de 1:3,209 (IC de 95\%: 1,772-5,814), tendo escore menor que 7 como referência. Para burnout com percepção de estresse $\geq 25$ pontos no PSS, foi estimada RP de 1:2,322 (IC de 95\%: 1,294-4,169), tendo como referência escores até 24. Foram encontrados valores FIV (Fator de Inflação da
Variância) abaixo de 10 e tolerâncias acima de 0,20, indicando não haver colinearidade.

A distribuição de respondentes segundo as variáveis independentes aparece na Tabela 2. Predominaram mulheres (81\%) em atividade na linha de frente. Foi informada idade igual ou inferior a 36 anos em 50\% das entrevistas, com média de 37,4 anos [desvio-padrão (dp): 9,4 anos], variando de 19 a 56 anos. Informaram residir em Novo Hamburgo 41\% do grupo. Informaram ter cônjuge 54\%, mas $87 \%$ disseram não viver sós. Foram identificadas, predominantemente, profissionais de nível técnico $(69 \%)$ e da área de enfermagem (76\%). Para 50\% do grupo, o tempo em atividade na profissão foi de oito anos ou mais, com média de nove anos (dp: 7,5 anos), variando de menos de 1 até 36 anos. $O$ tempo médio de atividade no hospital foi de 39,9 meses (dp: 46,1 meses), variando de menos de 1 mês até 20 anos completos, com mediana igual a 22 meses.

O estudo das cargas horárias de trabalho indica volumes elevados de atividade profissional pelas equipes. A carga total contratada teve mediana de 45 horas semanais e média de 51,8 horas (dp: 17,0 horas), variando de 30 a 96 horas semanais. A mediana do tempo efetivamente dedicado à linha de frente no hospital na semana anterior às entrevistas foi de 36 horas, com valor máximo referido de 84 horas, enquanto $24,4 \%$ do grupo referiu ter atuado nesse cenário por 45 horas ou mais naquela semana.

Exames para COVID-19 foram relatados por $68 \%$ do grupo; $25 \%$ referiram já ter tido resultado positivo em algum momento; e 15\% (19 profissionais) identificavam sintomas compatíveis com COVID-19 na entrevista. Entre sintomáticos na data, 4 (ou 21\% das pessoas com sintomas) nunca haviam sido testados ou estavam com exames encaminhados, mas ainda sem resultado.

Apenas 9\% do grupo informou estar em atendimento psicoterápico e 13\% estavam em uso de psicofármacos naquele momento. O uso eventual de bebidas alcoólicas foi referido em $41 \%$ das entrevistas e em nenhuma delas foi informado o uso de qualquer outra substância psicoativa, exceto álcool ou tabaco, este último referido por 10\% do grupo.

A Tabela 2 mostra, também, razões de prevalência e nível de significância (valores de p) para análises bivariáveis com variáveis independentes e cada um dos desfechos. Foram verificadas associações estatisticamente significativas entre o escore no SRQ-20 $\geq 7$ pontos e escolaridade de nível superior (RP: 1:0,436; IC de 95\%: 0,205-0,931), tendo nível técnico como referência, e também entre o escore no PSS $\geq 25$ pontos e idade $\geq 37$ anos completos (RP: 1:0,565; IC de 95\%: 0,327-0,975), tendo idade até 36 anos como referência.

A Tabela 3 apresenta as estimativas de razões de prevalência e intervalos de confiança obtidos nas análises ajustadas. Nenhuma associação estatisticamente significativa prevaleceu. 
Tabela 2. Distribuição dos profissionais segundo as covariáveis e razões de prevalência e valores de p para análises bivariáveis - Novo Hamburgo, 2020 (N = 123)

\begin{tabular}{|c|c|c|c|c|c|c|c|c|c|}
\hline \multirow{2}{*}{ Variável } & \multirow{2}{*}{ Categorias } & \multicolumn{2}{|c|}{ Distribuição } & \multicolumn{2}{|c|}{ SRQ-20 } & \multicolumn{2}{|c|}{ PSS } & \multicolumn{2}{|c|}{ OBI } \\
\hline & & $\mathbf{N}$ & $\%$ & $\geq 7 \mathrm{pts}$ & p Valor & $\geq 25$ & p Valor & Burnout & p Valor \\
\hline \multirow[t]{3}{*}{ Sexo } & & & & & 0,135 & & 0,239 & & 0,123 \\
\hline & Feminino & 100 & 81 & 1 & & 1 & & 1 & \\
\hline & Masculino & 23 & 19 & 0,494 & & 0,621 & & 0,483 & \\
\hline \multirow[t]{3}{*}{ Idade } & & & & & 0,133 & & 0,040 & & 0,430 \\
\hline & $\leq 36$ anos & 62 & 50 & 1 & & 1 & & 1 & \\
\hline & $\geq 37$ anos & 61 & 50 & 0,644 & & 0,565 & & 0,799 & \\
\hline \multicolumn{2}{|c|}{ Reside em Novo Hamburgo } & & & & 0,843 & & 0,741 & & 0,223 \\
\hline & Não & 72 & 59 & 1 & & 1 & & 1 & \\
\hline & $\operatorname{Sim}$ & 51 & 41 & 1,059 & & 0,913 & & 1,412 & \\
\hline \multirow[t]{3}{*}{ Tem cônju } & & & & & 0,319 & & 0,178 & & 0,173 \\
\hline & Não & 57 & 46 & 1 & & 1 & & 1 & \\
\hline & Sim & 66 & 54 & 0,704 & & 0,696 & & 0,679 & \\
\hline \multirow[t]{3}{*}{ Vive só } & & & & & 0,294 & & 0,776 & & 0,832 \\
\hline & Não & 107 & 87 & 1 & & 1 & & 1 & \\
\hline & Sim & 16 & 13 & 0,594 & & 1,115 & & 0,912 & \\
\hline \multirow[t]{3}{*}{ Escolarida } & & & & & 0,032 & & 0,342 & & 0,294 \\
\hline & Técnico & 85 & 69 & 1 & & 1 & & 1 & \\
\hline & Superior & 39 & 31 & 0,436 & & 0,746 & & 0,706 & \\
\hline \multicolumn{2}{|c|}{ Ingresso no estudo } & & & & 0,576 & & 0,725 & & 0,863 \\
\hline & SE 24 a 28 & 63 & 51 & 1 & & 1 & & 1 & \\
\hline & SE 29 a 33 & 60 & 49 & 1,008 & & 0,910 & & 1,050 & \\
\hline \multicolumn{2}{|c|}{ Atividade profissional } & & & & 0,392 & & 0,572 & & 0,552 \\
\hline & Demais áreas & 29 & 24 & 1 & & 1 & & 1 & \\
\hline & Enfermagem & 94 & 76 & 1,371 & & 0,843 & & 1,234 & \\
\hline \multicolumn{2}{|c|}{ Tempo de atividade profissional } & & & & 0,628 & & 0,289 & & 0,366 \\
\hline & Até 7 anos & 61 & 50 & 1 & & 1 & & 1 & \\
\hline & 8 anos ou mais & 62 & 50 & 0,870 & & 0,793 & & 0,773 & \\
\hline \multicolumn{2}{|c|}{ Tempo no HMNH } & & & & 0,442 & & 0,552 & & 0,534 \\
\hline & Até 22 meses & 62 & 50 & 1 & & 1 & & 1 & \\
\hline & 23 meses ou mais & 61 & 50 & 1,247 & & 1,173 & & 1,193 & \\
\hline Carga de & o segundo mediana & & & & 0,222 & & 0,459 & & 0,430 \\
\hline & $\leq 45 \mathrm{~h}$ semanais & 62 & 50 & 1 & & 1 & & 1 & \\
\hline & $\geq 46 \mathrm{~h}$ semanais & 61 & 50 & 0,701 & & 0,820 & & 0,799 & \\
\hline Carga en & de frente última semana & & & & 0,438 & & 0,197 & & 0,716 \\
\hline & $\leq 36 \mathrm{~h}$ semanais & 72 & 58 & 1 & & 1 & & 1 & \\
\hline & $\geq 37$ h semanais & 51 & 42 & 1,249 & & 1,412 & & 1,109 & \\
\hline Já foi test & a COVID-19 (qualquer exame) & & & & 0,437 & & 0,430 & & 0,728 \\
\hline & Não & 39 & 32 & 1 & & 1 & & 1 & \\
\hline & Sim & 84 & 68 & 1,286 & & 1,269 & & 0,901 & \\
\hline Exame pa & D-19 (qualquer) & & & & 0,259 & & 0,234 & & 0,663 \\
\hline & Negativo ou não fez & 92 & 75 & 1 & & 1 & & 1 & \\
\hline & Positivo & 31 & 25 & 1,512 & & 1,406 & & 1,167 & \\
\hline Com sintc & COVID-19 & & & & 0,084 & & 0,219 & & 0,375 \\
\hline & Não & 104 & 85 & 1 & & 1 & & 1 & \\
\hline & $\operatorname{Sim}$ & 19 & 15 & 1,775 & & 1,493 & & 1,368 & \\
\hline Em atend & psicoterápico & & & & 0,492 & & 0,638 & & 0,794 \\
\hline & Não & 112 & 91 & 1 & & 1 & & 1 & \\
\hline & $\operatorname{Sim}$ & 11 & 9 & 0,664 & & 0,783 & & 1,131 & \\
\hline Usa algun & ármaco & & & & 0,491 & & 0,776 & & 0,297 \\
\hline & Não & 107 & 87 & 1 & & 1 & & 1 & \\
\hline & Sim & 16 & 13 & 1,305 & & 1,115 & & 1,468 & \\
\hline Uso de ál & & & & & 0,127 & & 0,384 & & 0,177 \\
\hline & Não & 72 & 59 & 1 & & 1 & & 1 & \\
\hline & Sim & 51 & 41 & 0,623 & & 0,784 & & 0,664 & \\
\hline
\end{tabular}

SRQ-20: Self-Reporting Questionnaire; PSS: Perceived Stress Scale; OBI: Oldendurg Burnout Inventory. 
Tabela 3. Análises ajustadas por regressão de Poisson com variância robusta Novo Hamburgo, 2020 ( $N=123)$

\begin{tabular}{|c|c|c|c|c|c|c|c|}
\hline \multirow{2}{*}{ Variáveis } & \multirow{2}{*}{ Categorias } & \multicolumn{2}{|c|}{$S R Q-20 \geq 7$} & \multicolumn{2}{|c|}{ PSS $\geq 25$} & \multicolumn{2}{|c|}{ Burnout } \\
\hline & & $\mathbf{R P}$ & IC 95\% & RP & IC 95\% & $\mathbf{R P}$ & IC 95\% \\
\hline \multicolumn{8}{|c|}{ Demográficas } \\
\hline \multicolumn{8}{|l|}{ Sexo } \\
\hline & Feminino & 1 & & -- & & 1 & \\
\hline & Masculino & 0,626 & $0,238-1,653$ & -- & -- & 0,490 & $0,194-1,234$ \\
\hline \multicolumn{8}{|l|}{ Idade } \\
\hline & $\leq 36$ anos & 1 & & 1 & & -- & \\
\hline & $\geq 37$ anos & 0,634 & $0,357-1,128$ & 0,583 & 0,337-1,011 & -- & -- \\
\hline \multicolumn{8}{|c|}{ Tem cônjuge } \\
\hline & Não & -- & & 1 & & 1 & \\
\hline & Sim & -- & -- & 0,737 & $0,433-1,251$ & 0,687 & 0,393-1,201 \\
\hline \multicolumn{8}{|c|}{ Escolaridade } \\
\hline & Técnico & 1 & & -- & & -- & \\
\hline & Superior & 0,496 & $0,225-10,92$ & -- & -- & -- & -- \\
\hline
\end{tabular}

Relacionadas ao trabalho

Carga em linha de frente na última semana

\begin{tabular}{|c|c|}
\hline$\leq 36 \mathrm{~h}$ semanais & -- \\
\hline$\geq 37 \mathrm{~h}$ semanais & -- \\
\hline
\end{tabular}

Relacionadas à saúde

Com sintomas de COVID-19

$\begin{array}{lccc} & \text { Não } & 1 & \\ & \text { Sim } & 1,446 & 0,731-2,859\end{array}$

$\begin{array}{ccc}\text { Não } & 1 \\ \text { Sim } & 0,634 & 0,343-1,173\end{array}$

\begin{tabular}{|c|c|c|c|}
\hline -- & \multicolumn{3}{|c|}{1} \\
\hline-- & -- & 0,678 & $0,373-1,234$ \\
\hline
\end{tabular}

SRQ-20: Self-Reporting Questionnaire; PSS: Perceived Stress Scale; OBI: Oldendurg Burnout Inventory.

Os dados qualitativos parecem apontar na mesma direção dos achados quantitativos e reforçam a impressão de que condições específicas do contexto de enfrentamento à pandemia geram os efeitos psicossociais mensurados. Aparecem indícios de sofrimento psíquico nas entrevistas, como referências a estresse, medo e insegurança na linha de frente. Solicitações de indicações de serviços de atendimento emocional ou indicações do desejo de seguir nas entrevistas chegaram por meio da coleta de dados. Esse registro qualitativo reforça a constatação de elevado desgaste e sofrimento de quem atua em linha de frente.

"As equipes estão trabalhando em um estresse coletivo... Tem uma pressão diferente..." (Médica, 52 anos)

"Agora, nestes últimos dias, parece que triplicou minhas crises de ansiedade. Até to pensando em buscar uma ajuda profissional." (Enfermeira, 43 anos)

As manifestações de quem trabalha na linha de frente indicam forte nível de estresse, cansaço e muita dificuldade de lidar com o acréscimo de condições adversas que o enfrentamento da pandemia acarreta. $\mathrm{O}$ isolamento e o processo de trabalho aparecem nas falas como períodos de maior pressão e cansaço que o habitual. Longos plantões são usuais, mas agora piorados pela dificuldade de realizar intervalos, devido à paramentação, que precisa ser desfeita e refeita a cada saída da área reservada a pacientes COVID-19. Isso é compreendido como necessário, mas gerador de sobrecarga.

"...com suspeita de corona que teve uma parada cardíaca e o atendimento foi feito sem o uso correto de EPIs... Eu fui na cara e na coragem. Eu me arrisquei, depois eu pensei." (Técnica de enfermagem, 34 anos)

"Trabalho $12 \mathrm{~h}$ sem poder ir no banheiro, comer ou tomar água, porque não tem onde... é muita pressão o tempo todo." (Técnica de enfermagem, 32 anos)

O enfrentamento permanente com o risco da própria contaminação ganha destaque. Apareceram, ainda, ideias 
de culpa relacionadas às famílias, seja pela distância do isolamento ou pela proximidade na convivência, que aumenta a chance de contágio, já que estão atuando no cuidado com a saúde de pessoas contaminadas.

"Eu posso não saber que estou com esse vírus, então é um dilema, porque meu vô está doente e eu preciso ajudar, então ou eu ajudo a minha família ou me isolo." (Técnica de enfermagem, 32 anos)

A união da equipe foi evidenciada em todas as respostas como aspecto que favorece o desempenho.

"A ajuda da equipe diminui a exaustão. O pessoal, todo mundo se ajuda, tá todo mundo no mesmo barco..." (Técnica de enfermagem, 46 anos)

"Nos revezamos pra ver quem sai primeiro, quem fica de circulante fora, para as folgas..., revezamos pra não ficar tão pesado..." (Técnica de enfermagem, 26 anos)

\section{DISCUSSÃO}

Profissionais em atividade que responderam a este inquérito apresentavam evidências de sofrimento psíquico já nas entrevistas de ingresso no estudo, manifesto nos escores dos inventários SRQ-20, PSS e OBI, e qualificado em diferentes manifestações colhidas na análise qualitativa. A coleta de dados coincide com o pico da pandemia no município e no estado, então, a equipe do estudo encontra trabalhadores em meio ao incremento de demandas ${ }^{9,10}$. O prejuízo é abrangente e homogêneo entre aspectos distintos dos efeitos psicossociais da atividade sobre a saúde mental de profissionais ali expostos. A constatação de que os desfechos aparecem fortemente associados entre si e não associados a nenhuma das variáveis incluídas no modelo teórico é eloquente e sugestiva de que a sobrecarga na linha de frente da pandemia é a principal fonte de sofrimento. Estresse e esgotamento físico e emocional (burnout) não parecem modificados pelas demais condições estudadas, e os dados qualitativos oferecem elementos peculiares ao contexto da pandemia e percebidos por quem fazia os relatos como associados aos efeitos psicossociais manifestos.

Ambientes de trabalho em saúde costumam ser relacionados a algum grau de exposição a riscos e indução à sobrecarga emocional. Estudo transversal com profissionais de saúde em hospital universitário encontrou uma prevalência de $27,9 \%$ de escores elevados de SRQ-2017. Outro estudo, com 597 profissionais de saúde pública de Bento Gonçalves, RS, aplicou o SRQ-20 e encontrou 20,3\% dos profissionais com escore elevado. Nesse estudo, houve relação estatisticamente significativa com tempo de exercício profissional ( $p$ $=0,004)^{18}$, o que não verificamos em Novo Hamburgo. Esses estudos evidenciavam prevalências altas de escores elevados para o SRQ-20, mas ainda inferiores aos 40\% do grupo aqui entrevistado.
Revisando os dados obtidos com a PSS, entre outros grupos de profissionais da saúde ${ }^{20}$, também se verificam prevalências mais elevadas. Antes da pandemia, alguns estudos apresentavam resultados para a população geral, com predomínio de escores baixos ou moderados dos níveis de estresse, exceto para universitários da área da saúde que já tinham níveis moderado ou alto ${ }^{20}$. Profissionais da saúde, com mais vivência do setor e livres das especificidades da vida acadêmica, por outro lado, vinham apresentando níveis predominantemente baixo ou moderado de estresse ${ }^{21}$. Desde o início da pandemia, estudos com profissionais da saúde vêm demonstrando - corroborando os dados aqui apresentados - elevação das prevalências de níveis de estresse moderado ou alto, em grande parte relacionado a profissionais da linha de frente no combate à COVID-1922,23.

As entrevistas em profundidade também encontraram manifestações de sobrecarga e percepção de estresse, tensão e medo, e encontramos $45 \%$ do grupo com escores no PSS em níveis moderado, alto ou muito alto. O trabalho em linha de frente da COVID-19 envolve, em parte, formações de equipes para atuação em plantões e trabalho em contexto de emergência, mas caracteriza, em qualquer unidade especialmente destinada a esse enfrentamento, um trabalho em regime excepcional, com características muito peculiares. Falas dos entrevistados mencionaram que pressão e longas jornadas são costumeiros, mas há elementos adicionais no enfrentamento à COVID-19.

No Departamento de Emergência do Hospital da Universidade de Cork, na Irlanda, a equipe do setor era a mais suscetível ao burnout ${ }^{24}$. Foram acompanhados 95 profissionais por três meses, com inventários que incluíam o OBI. Três em cada quatro membros da equipe do departamento atingiram o ponto de corte para burnout.

Grandes epidemias desafiam a saúde mental dos profissionais da saúde, com demandas subitamente crescentes de pacientes com quadros graves. O sofrimento psicológico pode aparecer gradualmente na equipe, e sintomas de estresse pós-traumático aparecem mais tarde e duram muito tempo ${ }^{25,26}$. A resposta psicológica de trabalhadores da linha de frente na pandemia pode ser complexa e ainda não completamente delineada.

Um dos primeiros estudos publicados sobre saúde mental envolveu 994 profissionais de enfermagem e médicos da linha de frente em Wuhan, na China, com 22,4\% de indivíduos com distúrbios mentais moderados e 6,2\% com distúrbios graves². Outro estudo em Beijing, oito semanas após o surto epidêmico em Wuhan, encontrou prevalências de sintomas depressivos, ansiedade e estresse de 50,7\%, 44,7\% e $73,4 \%$, respectivamente ${ }^{27}$. Nossos achados foram coletados a partir da semana de início do aumento de número de infectados na região e ao longo do período que correspondeu ao pico da epidemia na região onde está situado o hospital, e a percepção de estresse também foi considerada elevada. 
Com o avanço no número de casos, estudos de diferentes países têm replicado esses achados ${ }^{28-31}$. Os achados aqui apresentados corroboram os descritos por esses e por outros estudos ${ }^{32-34}$, em que profissionais da linha de frente mostraram maior frequência de sinais de exaustão e burnout em diferentes momentos da evolução da pandemia.

As condições destacadas nas entrevistas em profundidade como estressantes e a referência a cansaço, estresse e sofrimento acompanharam os dados obtidos com a aplicação dos inventários propostos (SRQ-20, PSS e OBI). Longas jornadas e dificuldades com a paramentação, entre outros itens agravantes do estresse, não estavam representadas nos itens investigados nas entrevistas da dimensão quantitativa, assim como a união da equipe e o suporte mútuo mencionados como atenuantes do sofrimento também não estavam. Investigações futuras poderão considerar esses achados e, talvez, contemplar tais variáveis. Os achados deste estudo corroboram indicações de cuidados com as equipes, como: repouso e intervalos com escalas diferenciadas, adequações de rotinas e espaços físicos, além da oferta de apoio emocional às equipes ${ }^{27-34}$.

Mesmo não modificando os desfechos do estudo, o achado de $68 \%$ de profissionais testados e $25 \%$ com resultado positivo para COVID-19 parece elevado se comparado à população geral ${ }^{10}$. Profissionais de saúde estão, de fato, mais expostos. Como o resultado negativo mantém a possibilidade do contágio e todas as expectativas inerentes, apareceram muitas falas indicando preocupação persistente com a própria contaminação ou a transmissão do vírus a terceiros. Testagem não parece amenizar os efeitos psicossociais da exposição.

São limitações do estudo o tamanho da amostra e seu caráter local. O poder estatístico é limitado pelo tamanho reduzido da amostra $(n=123)$. Não se pode afastar a possibilidade de erro tipo II. Como o enfrentamento à COVID-19 promove cenários totalmente novos e qualquer informação nesse contexto pode ganhar relevância ao viabilizar cotejamento com estudos semelhantes, parece válida a exposição desses dados.

\section{CONCLUSÕES}

São elevadas as prevalências de sofrimento psíquico, estresse percebido e burnout e suas dimensões nesse grupo de profissionais em atividade na linha de frente da pandemia. Os dados qualitativos e o achado casual da rotatividade nas equipes, com grande número de profissionais já desligados quando procurados pelos pesquisadores, reforçam esse achado e o qualificam. As entrevistas em profundidade oferecem novas indicações de elementos especificamente relacionados à atividade a serem avaliados em estudos futuros.
Os pedidos de indicação de atendimento também reforçam a percepção de sobrecarga. Inicialmente, recomenda-se priorizar repouso e intervalos com escalas diferenciadas, na medida do possível, o que poderá exigir adequações de rotinas e espaços físicos, além de ampliar a oferta de apoio emocional às equipes.

\section{CONTRIBUIÇÕES INDIVIDUAIS}

Rogério Lessa Horta - Concepção e projeto, coordenação do campo, análise e interpretação dos dados, redação do artigo, aprovação final da versão a ser publicada; responsável por todos os aspectos do trabalho.

Eduardo Guimarães Camargo - Concepção e projeto, coordenação do campo, aprovação final da versão a ser publicada; responsável por todos os aspectos do trabalho.

Marcus Levi Lopes Barbosa - Coordenação do campo, análise e interpretação dos dados, aprovação final da versão a ser publicada.

Pedro José Sartorelli Lantin - Concepção e projeto, coordenação do campo, redação do artigo, aprovação final da versão a ser publicada.

Talia Greici Sette - Coordenação do campo, redação do artigo, aprovação final da versão a ser publicada.

Thaís Caroline Guedes Lucini - Redação do artigo, aprovação final da versão a ser publicada.

Aline Faria Silveira - Redação do artigo, aprovação final da versão a ser publicada.

Lizziê Zanini - Redação do artigo, aprovação final da versão a ser publicada.

Bibiana Andrade Lutzky - Redação do artigo, aprovação final da versão a ser publicada.

\section{CONFLITO DE INTERESSES}

Nenhum dos autores tem qualquer conflito de interesse a destacar.

\section{AGRADECIMENTOS}

Agradecemos, pela acolhida e o apoio, as instâncias diretivas e todo o corpo técnico da Fundação de Saúde de Novo Hamburgo e do Hospital Municipal de Novo Hamburgo, sem as quais este trabalho não teria sido possível.

\section{REFERÊNCIAS}

1. Dong L, Bouey J. Public Mental Health Crisis during COVID-19 Pandemic, China. Emerg Infect Dis. 2020;26(7):1616-8. 
2. Kang L, Ma S, Chen M, Yang J, Wang Y, Li R, et al. Impact on mental health and perceptions of psychological care among medical and nursing staff in Wuhan during the 2019 novel coronavirus disease outbreak: A cross-sectional study. Brain Behav Immun. 2020;87:11-7.

3. Lai J, Ma S, Wang Y, Cai Z, Hu J, Wei N, et al. Factors Associated With Mental Health Outcomes Among Health Care Workers Exposed to Coronavirus Disease 2019. JAMA Netw Open. 2020;3(3):e203976.

4. Pfefferbaum B, North CS. Mental Health and the Covid-19 Pandemic. N Engl J Med. 2020;383:510-2

5. Chung JPY, Yeung WS. Staff Mental Health Self-Assessment During the COVID-19 Outbreak. East Asian Arch Psychiatry. 2020;30(1):34.

6. Chen $Q$, Liang M, Li Y, Guo J, Fei D, Wang L, et al. Mental health care for medical staff in China during the COVID-19 outbreak. Lancet Psychiatry. 2020;7(4):e15-6.

7. Greenberg N, Docherty M, Gnanapragasam S, Wessely S. Managing mental health challenges faced by healthcare workers during covid-19 pandemic. BMJ. 2020;368:m1211.

8. Ho CS, Chee CY, Ho RC. Mental Health Strategies to Combat the Psychological Impact of COVID-19 Beyond Paranoia and Panic. Ann Acad Med Singapore. 2020;49(3):1-3.

9. Rio Grande do Sul. Governo do Estado. Centro de Operações de Emergência do Rio Grande do Sul/COERS [Internet]. Boletim epidemiológico COVID-2019-SE, de 24 de julho de 2020. Disponível em: https://coronavirusadmin.rs.gov.br/upload/arquivos/202006/18134431be-covid-19-coers-se-24-160620.pdf. Acesso em: 29 jul. 2020.

10. Rio Grande do Sul. Governo do Estado. Centro de Operações de Emergência do Rio Grande do Sul/COERS [Internet]. Boletim epidemiológico COVID-2019-SE, de 19 de agosto de 2020. Disponível em: https://coronavirus.rs.gov.br/upload/arquivos/202008/19094008be-covid19-resumido-se-33.pdf. Acesso em: 26 ago. 2020.

11. Gonçalves DM, Stein AT, Kapczinski F. Avaliação de desempenho do Self-Reporting Questionnaire como instrumento de rastreamento psiquiátrico: um estudo comparativo com 0 Structured Clinical Interview for DSM-IV-TR. Cad. Saúde Pública. 2008;24:380-90.

12. Luft CDB, Sanches SO, Mazo GZ, Andrade A. Versão brasileira da Escala de Estresse Percebido: tradução e validação para idosos. Rev Saúde Pública. 2007;41(4):606-15.

13. Faro A. Análise fatorial confirmatória das três versões da Perceived Stress Scale (PSS): um estudo populacional. Psicol Reflex Crít. 2015;28(1):21-30.

14. Schuster MS, Dias VV. Oldenburg Burnout Inventory - validação de uma nova forma de mensurar Burnout no Brasil. Ciênc Saúde Coletiva. 2018;23(2):553-62.

15. Bauer WM, Gaskell G. Pesquisa qualitativa com texto, imagem e som. 7a ed. Petrópolis: Vozes; 2008.

16. Creswell JW. Projeto de Pesquisa: métodos qualitativo, quantitativo e misto. 3 a ed. Porto Alegre: Artmed; 2010.

17. Ebling M, Carlotto MS. Burnout syndrome and associated factors among health professionals of a public hospital. Trends Psychiatry Psychother. 2012;34(2):93-100.

18. Faria NMX, Klosinski RFS, Rustick G, Oliveira LM. Mental health of public health workers in Bento Gonçalves, Rio Grande do Sul, Brazil. Rev Bras Med Trab. 2018;16(2):145-57.

19. Teixeira FD, Prebianchi HB. Comprometimento, estresse e satisfação com a vida de profissionais da saúde. Rev Psicol Organ Trab. 2019;19(2):598-606.
20. Yosetake AL, Camargo IML, Luchesi LB, Gherardi-Donato ECS, Teixeira CAB. Estresse percebido em graduandos de enfermagem. SMAD, Rev Eletrônica Saúde Mental Álcool Drog (Ed Port). 2018;14(2):117-24.

21. Zhan Y, Liu Y, Liu H, Li M, Shen Y, Gui L, Zhang J, et al. Factors associated with insomnia among Chinese front-line nurses fighting against COVID-19 in Wuhan: A cross-sectional survey. J Nurs Manag. 2020;28(7):1525-35.

22. Xiao X, Zhu X, Fu S, Hu Y, Li X, Xiao J. Psychological impact of healthcare workers in China during COVID-19 pneumonia epidemic: A multi-center cross-sectional survey investigation. J Affect Disord Rep. 2020;274:405-10.

23. O'Kelly F, Sparks S, Seideman C, Gargollo P, Gramberg C, Ko J, et al. A survey and panel discussion of the effects of the COVID-19 pandemic on paediatric urological productivity, guideline adherence and provider stress. J Pediatr Urol. 2020;16(4):492.e1-492.e9.

24. Chernoff P, Adedokum C, O'Sullivan L, Mcmanus J, Payne A. Burnout in the Emergency Department hospital staff at Cork University Hospital. Ir J Med Sci. 2019;188(2):667-74.

25. Verma S, Mythily S, Chan YH, Deslypere JP, Teo EK, Chong SA. Post-SARS psychological morbidity and stigma among general practitioners and traditional Chinese medicine practitioners in Singapore. Ann Acad Med Singapore. 2004;33(6):743-8.

26. Wu P, Fang Y, Guan Z, Fan B, Kong J, Yao Z, et al. The psychological impact of the SARS epidemic on hospital employees in China: exposure, risk perception, and altruistic acceptance of risk. Can J Psychiatry. 2009;54(5):302-11.

27. Zhang C, Yang L, Liu S, Ma S, Wang Y, Cai Z, et al. Survey of insomnia and related social psychological factors among medical staff involved in the 2019 novel coronavirus disease outbreak. Front Psychiatry. 2020;11:306.

28. Tan BYQ, Chew NWS, Lee GKH, Jing M, Goh Y, Yeo LLL, et al. Psychological Impact of the COVID-19 Pandemic on Health Care Workers in Singapore. Ann Intern Med. 2020;173(4):317-20.

29. Bohlken J, Schömig F, Lemke MR, Pumberger M, Riedel-Heller SG. COVID-19 pandemic: stress experience of healthcare workers - a short current review. Psychiatr Prax. 2020;47:190-7.

30. Evanoff BA, Strickland JR, Dale AM, Hayibor L, Page E, Duncan JG, et al. Work-related and Personal Factors Associated with Mental Well-being during COVID-19 Response: A Survey of Health Care and Other Workers. J Med Internet Res. 2020;22(8):e21366.

31. Rossi R, Socci V, Pacitti F, Di Lorenzo G, Di Marco A, Sicarusano A, et al. Mental Health Outcomes Among Frontline and Second-Line Health Care Workers During the Coronavirus Disease 2019 (COVID-19) Pandemic in Italy. JAMA Netw Open. 2020;(5):e2010185.

32. Sasangohar F, Jones SL, Masud FN, Vahidy FS, Kash BA. Provider Burnout and Fatigue During the COVID-19 Pandemic: Lessons Learned From a High-Volume Intensive Care Unit. Anesth Analg. 2020;131(1):106-11.

33. Barello S, Palamenghi L, Graffigna G. Burnout and somatic symptoms among frontline healthcare professionals at the peak of the Italian COVID-19 pandemic. Psychiatry Res. 2020;290:113129.

34. Hu D, Kong Y, Li W, Han Q, Zhang X, Zhu LX, et al. Frontline nurses' burnout, anxiety, depression, and fear statuses and their associated factors during the COVID-19 outbreak in Wuhan, China: A large-scale cross-sectional study. EClinicalMedicine. 2020;24:100424. 